\title{
Autonomous parametric process for daylight simulation applied to the proposal of a daylighting of buildings performance tool
}

XXIV International Conference of the Iberoamerican Society

\author{
Pedro Oscar Pizzetti Mariano \\ Universidade Federal de Santa Catarina | Brasil | pedro.pm@hotmail.com \\ Raphaela Walger da Fonseca \\ Universidade Federal de Santa Catarina | Brasil | ruttkay.pereira@ufsc.br \\ Fernando Oscar Ruttkay Pereira \\ Universidade Federal de Santa Catarina | Brasil | raphawf@gmail.com
}

Alice Theresinha Cybis Pereira

Universidade Federal de Santa Catarina | Brasil | alice.cybis.pereira@ufsc.br

\begin{abstract}
The openings features definition, considering the obstructions influence caused by the urban environment, are extremely relevant for the daylit buildings design. The complexity of the daylight phenomenon and the need to estimate its performance spread the use of parametric simulation and simulation programs. Thus, this article aims to create a parametric process, derived from a digital process, capable of simulating and registering the performance of daytime construction in different urban scenarios in an automated way. This process made it possible to generate a series of data capable of producing tools for understanding the phenomenon of natural daylight.
\end{abstract}

Keywords: Parametric process; Simulation; Daylighting; Building performance.

\section{INTRODUÇÃO}

O dimensionamento das aberturas e a definição das características arquitetônicas dos ambientes são de suma importância para o desenvolvimento do projeto de edificações que visa o aproveitamento da luz do dia. Ambos influenciam seu resultado tanto no aspecto formal, quanto performático. A interpretação do fenômeno da luz natural associada a otimização dos projetos, é convertida em benefícios aos usuários. Os resultados projetuais consequentes da busca pela iluminação natural ideal (sem a presença de ofuscamento ou o desconforto térmico) podem ser interpretadas de modo dispare por distintos profissionais e áreas, tais como arquitetura, economia, custo, gerenciamento de carga, consumo de energia, entre outros. Podendo-se também variar por fenômenos externos aos envolvidos em sua análise como localidade geográfica, cultura e momento econômico (ALSHOUBAKI et al., 2016; BOYCE ,1996).

Independentemente das inúmeras interpretações e indicadores para caracterizar o a qualidade do ambiente luminoso, há consenso que os usuários apreciem locais onde as aberturas proporcionem acesso à luz natural, possibilitando contato visual com o ambiente externo, sem a presença de ofuscamento (REINHART, MARDALJEVIC e ROGERS, 2006; WIRZ-JUSTICE, 2010).

A complexidade do fenômeno e a necessidade de conhecer o desempenho luminoso das edificações faz com que diferentes programas de simulação sejam desenvolvidos e empregados durante o processo de projeto. Na busca por uma edificação bem iluminada naturalmente, é importante que se possa testar diversas alternativas projetuais. Dentre as complexidades da caracterização do ambiente luminoso estão a sazonalidade do clima, e as variações de disponibilidade de luz ao longo do ano e do dia, a relação da edificação com o seu entorno imediato, natural ou construído, além das características arquitetônicas da própria edificação. Nesse sentido, a modelagem e a simulação paramétrica permitem alterações rápidas e geram pré-visualizações dos resultados, auxiliando na compreensão e tomadas de decisões (FELIPPE et al., 2015; CARTANA, PEREIRA e BERTE, 2016)

Os recentes avanços na ciência da computação e as exigências de comprovação do desempenho das edificações impulsionaram o desenvolvimento de métodos de simulação. Para isso, adota-se uma abordagem integrada de sistemas generativos, processos de parametrização e otimização. A introdução de algoritmos de parametrização, aliados a ferramentas computacionais no processo projetual, permite a rápida exploração de grande quantidade de alternativas, aumentando as possibilidades de investigação de diferentes variáveis, para avaliações de diversos fenômenos relacionados a iluminação natural (FELIPPE et al., 2015; JAKUBIEC e REINHART, 2011). Esses avanços são especialmente úteis no contexto atual em que a iluminação natural é usualmente avaliada com base em dados climáticos, o que exige grande esforço computacional. Tais avaliações, denominadas "climate based daylight modelling" (CBDM) são realizadas, geralmente, para um ano inteiro, em um intervalo de tempo de uma hora ou menos, a fim de 


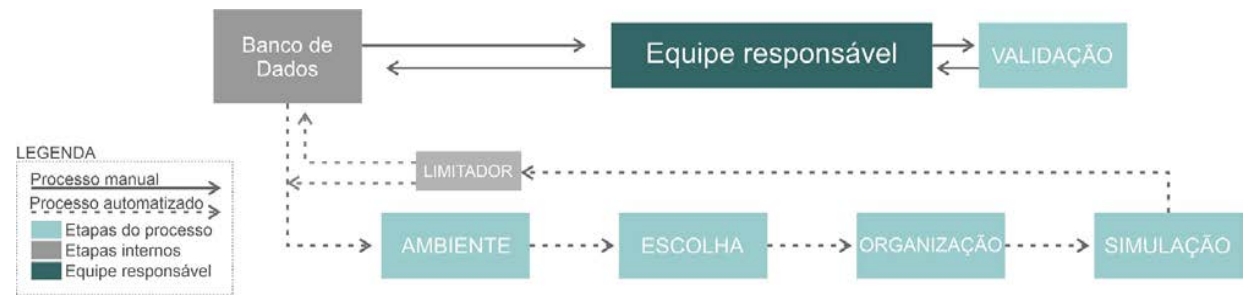

Figura 1: Estrutura lógica do processo paramétrico, Fonte: Os autores (2020)

capturar a dinâmica diária e sazonal da luz natural do dia. (MARDALJEVIC e CHRISTOFFERSEN, 2017).

No entanto, mesmo com as facilidades provenientes dos processos paramétricos, em alguns casos, estes processos demandam ações repetitivas, consumindo um demasiado tempo que poderia ser disponibilizado para a análise e aplicação de seus resultados. Diante disso, o presente trabalho busca criar um processo paramétrico de simulação capaz de avaliar e registrar o comportamento da luz natural em diferentes cenários urbanos e ambientes internos, de modo automatizado.

\section{METODOLOGIA E DESENVOLVIMENTO DA PROGRAMAÇÃO PARAMÉTRICA}

O processo paramétrico descrito nesse artigo busca, de forma automatizada, captar informações préestabelecidas, transformá-las em um modelo geométrico tridimensional digital, executar simulações da iluminação natural diurna e coletar e organizar seus resultados.

Para o desenvolvimento da programação foi utilizado uma estrutura teórica adaptada dos estudos de Oxman (2005 e 2006) e Oxman e Hammer (2007) o "Performance Model", variando as ações, mas mantendo seu desenvolvimento cíclico. Esse modelo foi escolhido pois permite variações e modificações e é indicado para propostas em que a importância do desenvolvimento da forma e da performance do ambiente são equivalentes. A variação proposta empregou os conceitos já utilizados nos trabalhos de Mariano (2017, 2018), Ambiente, Escolha, Organização e Simulação, em efeito cascata, no qual uma ação só se inicia quando a anterior está completa. Esses elementos foram inseridos dentro da estrutura de Oxman mantendo sua etapa de validação, mas deslocando-a para o fim do processo. Com a compatibilização das estruturas teóricas o processo paramétrico se consolidou em 5 passos, quatro para o funcionamento da programação e um para a validação de seus resultados. A estrutura lógica do trabalho pode ser interpretada pela figura 1.

Cada uma dessas etapas obedece a uma função e funciona à medida que, em sequência, sejam desempenhadas. Na programação desenvolvida para esse artigo as etapas cumprem a função de: Ambiente modela o entorno urbano e o ambiente da edificação a ter seu desempenho simulado -; Escolha - caracteriza atributos ao ambiente de simulação previamente definidos; - Organização - compõe e organiza os elementos geométricos e não geométricos (informações)-; Simulação - o fenômeno da iluminação natural é simulado para o período de um ano; e Validação - com os diferentes dados gerados (numéricos e visuais) uma validação não autônoma é feita interpretando os resultados.

Para este processo paramétrico as quatro primeiras etapas têm seu processo automatizado, coletando e gerando resultados de modo cíclico e autônomo, por meio de um banco de dados e um limitador pré-estabelecido. A última etapa, a validação, não apresenta essas características, pois acontece após a finalização de um número prédeterminado de ciclos. E é a análise dos resultados, por um grupo de especialistas ou desenvolvedores, que pode validar ou não o processo e tomar decisões para novos ciclos autônomos ou correções.

\section{PROGRAMAÇÃO VISUAL PARAMÉTRICA AUTÔNOMA}

O processo paramétrico de simulação para avaliação da iluminação natural diurna utilizou o Software Rhinoceros 3D-6 (MCNEE, 2014), seu componente Grasshopper (Rutten e McNeel \& Associates, 2004) e plug-ins DIVA (SOLEMMA, 2014), Ladybug (Sadeghipour Roudsari e Pak, 2013). Esse conjunto de ferramentas digitais foi escolhido pois segundo Emami, Khodadadi e Von Buelow (2014) possui alta capacidade de performance utilizando as estruturas do RADIANCE (WARD, 1994). Também foram utilizados o programa Excel 2016 para a descrição das características geométricas e não geométricas das simulações, além de um plug-in para ações iteradas o Hoopsnack.

O processo da construção da programação iniciou com um apanhado teórico sobre o assunto sequenciado pelo desenvolvimento do algoritmo visual em 5 estágios, sendo: a formação de um ambiente para simulação; a verificação do ambiente e a inserção de informações no modelo; os ajustes e combinações; as simulações e coleta dos dados; e por fim os verificações e validações.

Essas etapas seguiram um conjunto de parâmetros que foram decodificados pela programação paramétrica, com o objetivo de se obter diferentes dados de saída. As características que o modelo pode reproduzir e alguns dos alvos não são concentradas somente em uma das etapas, mas sim organizados visando a automatização do processo. A seguir, na tabela 1, é apresentado um resumo de todos os parâmetros. Suas particularidades e posicionamento são destrinchadas no decorrer da estruturação da metodologia. 


\begin{tabular}{|c|c|c|}
\hline Parâmetros & \multicolumn{2}{|c|}{ Valores } \\
\hline \multicolumn{3}{|l|}{ Ambiente } \\
\hline Pé-direito & $2,7 \mathrm{~m}$ & \\
\hline Altura da verga (h) & $2,1 \mathrm{~m}$ & \\
\hline Largura (I) & $1,5 \mathrm{~m}$ & $4,5 \mathrm{~m}$ \\
\hline Profundidade & $\left.\right|^{*} 1,5$ & $l^{*} 2,5$ \\
\hline $\begin{array}{l}\text { Refletância das } \\
\text { superfícies }\end{array}$ & \multicolumn{2}{|c|}{ Piso: $20 \%$; Parede: $50 \%$; Teto: $80 \%$} \\
\hline Orientação & Norte & Oeste \\
\hline \multicolumn{3}{|l|}{ Abertura } \\
\hline Área & \multicolumn{2}{|l|}{ 1/6 da área do piso } \\
\hline $\begin{array}{l}\text { Transmissão } \\
\text { visível do vidro }\end{array}$ & $88 \%$ & $40 \%$ \\
\hline $\begin{array}{l}\text { Localização da } \\
\text { abertura }\end{array}$ & $\begin{array}{l}\text { Centro do } \\
\text { ambiente }\end{array}$ & $\begin{array}{l}\text { Alinhada à uma } \\
\text { das laterais do } \\
\text { ambiente (canto) }\end{array}$ \\
\hline Peitoril janela & inexistente & existente \\
\hline
\end{tabular}

\begin{tabular}{lll}
\hline Sacada & & \\
\hline Condição & inexistente & existente \\
Profundidade & - & $1,5 \mathrm{~m} \quad 3 \mathrm{~m}$ \\
\hline Peitoril da sacada & inexistente & existente \\
\hline $\begin{array}{ll}\text { Refletância } \\
\text { superfícies }\end{array}$ & Piso: 20\%; Parede: 50\%; Teto: $80 \%$ \\
\hline
\end{tabular}

\begin{tabular}{|c|c|}
\hline \multicolumn{2}{|l|}{ Entorno Urbano } \\
\hline $\begin{array}{l}\text { Angulos de } \\
\text { Obstrução do } \\
\text { entorno }\end{array}$ & $\begin{array}{l}20 \% ; 30 \% ; 40 \% ; 45 \% ; 55 \% ; 60 \% ; 65 \% \\
70 \% ; 80 \%\end{array}$ \\
\hline $\begin{array}{l}\text { Refletância do } \\
\text { entorno }\end{array}$ & $\begin{array}{l}\text { Piso externo: } \\
10 \%\end{array}$ \\
\hline
\end{tabular}

Tabela 1: Resumo dos parâmetros utilizados no processo.

O processo inicia com o desenvolvimento da etapa Ambiente. Nessa etapa, um ambiente urbano e um residencial são modelados a partir de duas fontes de informações, a primeira pré-estabelecida na interface de paramétrica (Grasshopper) por meio de parâmetros ajustáveis em "sliders" e a segunda por tabelas feitas no Excel que são lidas pelo processo de forma autônoma. Cada uma dessas fontes de informações permite construir diferentes características do entorno urbano. Como observado pela figura 2 .

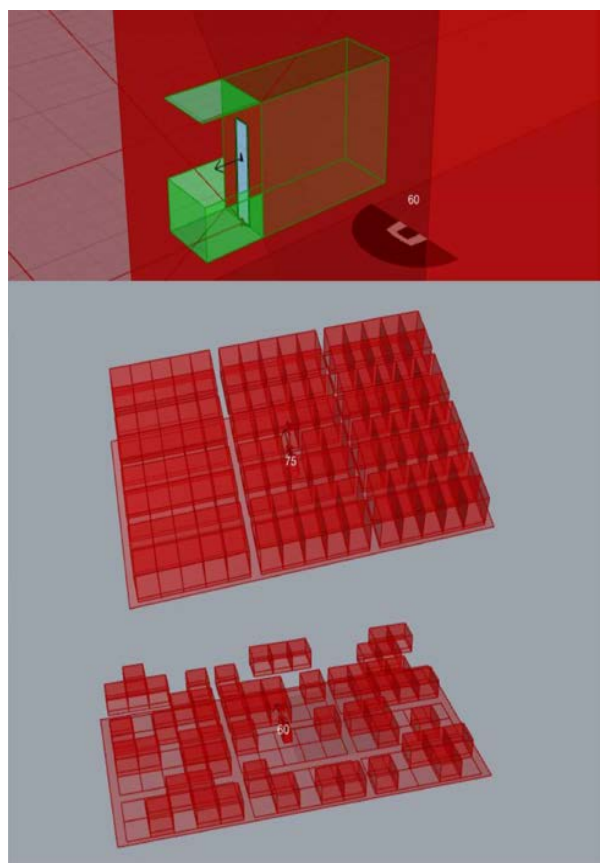

Figura 2: Exemplos da leitura dos parâmetros da programação para a etapa Ambiente.

O ambiente de simulação trata-se de um ambiente genérico de uma unidade habitacional, de um edifício multifamiliar hipotético. $\mathrm{O}$ ambiente base possui geometria regular cuja a planta varia do formato de um quadrado para um retângulo. Foi considerada a presença de uma abertura para o exterior com acesso à luz natural. Os parâmetros variados no Grasshopper foram: comprimento e largura das quadras; altura do ambiente de análise (pé direito); altura do peitoril da janela; altura da verga da janela; altura do modelo de simulação em relação ao solo (pavimento); dimensões do edifício que envolve o ambiente de simulação; raio do entorno a ser analisado; lotes ocupados no raio do entorno escolhido (escolhidos por meio de uma porcentagem de lotes); a possibilidade de escolher variações randômicas dos lotes ocupados (exemplificada pelo quadro final da figura 2); e divisão de lotes por quadra.

Os parâmetros escolhidos no Excel são divididos em dois grupos, em números racionais e números inteiros (códigos). Os números racionais são: largura do ambiente a ser analisado; profundidade do ambiente; profundidade da sacada; taxa de ocupação dos edifícios do entorno urbano; e ângulo de obstrução horizontal. Os em números inteiros são: o tipo de abertura (booleana, o ou 1); tipo de guarda corpo da sacada (booleana, o ou 1); orientação $(0=$ Sul, 1 = Leste, 2 = Oeste e 3 = Norte); e localização geográfica ( 0 = Belém; 1 = Brasília e 3 = Curitiba).

Os parâmetros globais do modelo, os que correspondem aos aspectos da entorno urbano e ambiente de simulação, podem ser alterados para serem utilizados em outros estudos. Os parâmetros mais específicos como a localização, horário de simulação e propriedades ópticas da superfície, necessitam de modificações específicas na 
programação, como a adição de arquivos externos ou alterações de componentes internos do DIVA. Essas modificações podem ser executadas na etapa seguinte da programação.

Para a segunda etapa, Escolha, são escolhidas as características que o modelo digital utiliza para fazer as simulações. Esses atributos são voltados ao cálculo do comportamento da luz natural como reflexões, tamanho da malha de análises, posição dos pontos para orientação da máscara de sombra, inserção de novos arquivos climáticos, escolha do intervalo de tempo de análise e nomeação de como os arquivos (imagens e planilhas) serão salvos.

Com esses atributos designados a programação continua com sua etapa de Organização separando as características geométricas e não geométricas, nomeando e designando onde serão salvos (dados, planilhas e imagens). As características são organizadas em duas pilhas de simulação, a primeira do plug-in DIVA ("Annual Daylight" e "Daylight Factor", para simulações dinâmicas) e outra do plug-in LadyBug (Ladybug_shadingMask). Na pilha do plug-in Diva é possível escolher a iluminância alvo, a porcentagem do tempo que ela ocorre. Na saída dessa pilha foram organizados 8 espaços de coleta, podendo identificar 4 alvos diferentes, além de analisar o resultado com ou sem a presença da sacada. Na pilha do Ladybug outros 2 espaços de coleta de dados foram separados, um para a porcentagem de céu visível e outra para o céu obstruído. Todos os dados numéricos de saída são direcionados para a mesma tabela que fornece os dados em uma aba separada. Esses dados podem ser considerados como os dados de saída do processo (os "outputs"), dentro da etapa de simulação.

Na etapa seguinte, Simulação, o dispositivo que permite as repetições das simulações é inserido e ajustado. Para essa função utilizou-se a pilha Hoopsnack, que permite a repetição de ações iteradas, admitindo que as informações presentes na planilha sejam captadas e transmitidas para a programação repetidas vezes sem que seja necessário a interferência manual. A pilha foi programada para funcionar de três formas, decodificando e simulando somente um único caso; decodificando e simulando um conjunto de casos; ou lendo, por completo, os casos presentes na planilha e simulando-os. Essa última função apresenta variações, podendo-se designar um limitador para que não simular todos os itens, além de permitir a escolha de um ponto de partida. Assim é possível pausar e retomar as simulações caso seja necessário. Esse conjunto de ações é apresentado pela figura 3.

Com a etapa de Simulação as quatro partes que compõe o processo digital estão completas, contudo, para que o processo funcione é necessário a quinta etapa a Validação. Essa última estrutura é utilizada para que, antes e depois de uma rodada de simulação, os parâmetros descritos nas planilhas sejam organizados, e ao fim do processo, possa-se avaliar se as simulações obtiveram êxito, ou se é necessário nova rodadas de teste e modificações.

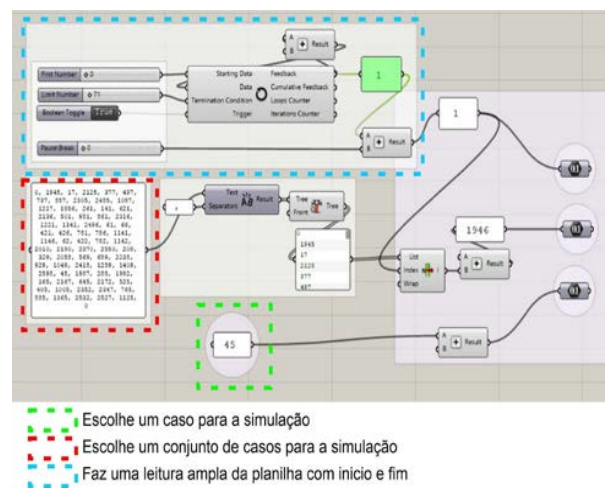

Figura 3: As três formas programadas para a leitura dos casos a serem simulados.

\section{ETAPA DE TESTES}

Para a verificação e validação dessas etapas, diversos testes foram feitos, possibilitando ajustes e adições de elementos às etapas da programação apresentada. Estas avaliações para a validação do modelo antes de seu uso foram feitas durante a etapa de Validação. A validação consistiu na análise crítica dos resultados de lotes de simulação por um grupo de 6 especialistas na área de iluminação natural e pela comparação de resultados de alguns casos selecionados com simulações feitas diretamente no programa de simulação. No primeiro caso, diversos lotes foram simulados progressivamente, de acordo com o feedback dos especialistas. Sendo que os três maiores testes contaram com mais de 1500 casos cada, antes do modelo paramétrico ser validado. No segundo caso, a cada etapa de testes foram escolhidos alguns casos críticos para serem modelados diretamente no programa Rhinoceros e simulados no DIVA por um membro do grupo de especialistas, para a aferição dos resultados.

Como exemplo das verificações feitas na etapa de avaliação, cita-se a correção de um erro associado à modelagem (etapa Ambiente). Tal erro resultava em distorções na contabilização do efeito da obstrução da luz causada por edificações do entorno, bem como da sua reflexão no entorno imediato ao ambiente analisado.

\section{RESULTADOS}

Como resultado da aplicação do processo paramétrico, foram simulados nove pacotes, com 2640 ambientes cada, por duas vezes. A aplicação do processo permitiu que a interação humana fosse feita somente nos momentos de ajustes das informações, definido os parâmetros e casos a serem simulados, e ao fim de cada grupo, verificando os resultados. A figura 4 apresenta a programação inteira. Por meio da figura é possível observar a localização de cada uma das etapas descritas durante a criação da estrutura do processo paramétrico. 


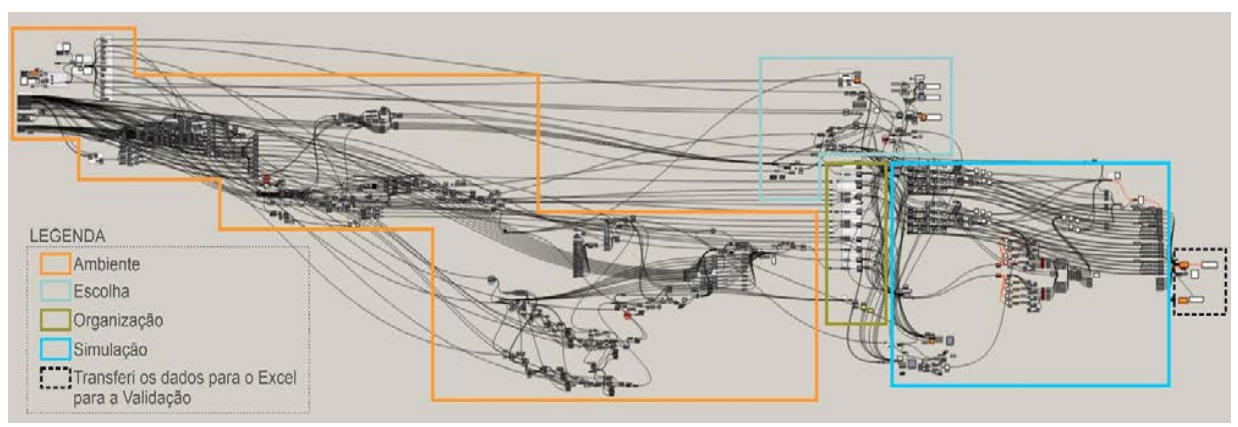

Figura 4: llustração das etapas setorizadas descritas na formação do processo paramétrico

Para cada simulação feita, uma série de dados e imagens eram organizadas e salvas. Os arquivos que continham as informações da simulação foram guardados em uma pasta do programa DIVA, com um nome único (exemplo: SIMXXXX_80tv_UNI_CURI_Simulation_Annuanl_Daylight ). As duas imagens, referentes aos mapas de autonomia de luz natural e das máscaras da sombra, foram salvas nas pastas designadas na etapa Organização. Os dados numéricos dos resultados foram salvos no mesmo arquivo do Excel que continha as informações de entrada, mas em uma nova aba. A figura 5 apresenta os resultados do processo.

O programa DIVA for-Rhino separa a simulação em dois momentos. A primeira, na qual se obtém o "daylight" coeficientes (TREGENZA e WATERS, 1983) e se processa a simulação anual propriamente dita e, a segunda, em que se aplicam os filtros de iluminância alvo para a estimativa da autonomia da luz natural. A primeira parte da simulação é significativamente mais demorada podendo levar em média $90 \%$ do tempo total do processo. A segunda parte é consideravelmente menor, pois utiliza os dados da primeira, mas os organiza conforme um filtro parametrizado. A nomeação do arquivo para os dados da simulação tem importância ao se requisitar novas informações de saída, como diferentes alvos de iluminância para a autonomia da luz natural, sem a necessidade de realizar a primeira parte da simulação novamente. Assim, os resultados coletados pela programação foram utilizados para a obtenção da autonomia da luz natural para em dois momentos. Inicialmente, foi identificando o comportamento da luz com alvos maiores (300, 250, 200 e 100) e, na sequência, com menores (90, 80, 70 e 60$)$. A repetição da segunda parte da simulação, com parâmetros de saída diferenciados, possibilitou ampliar o número de resultados finais de maneira otimizando, poupando-se tempo de simulação.

Ao final, foram coletadas as informações da porcentagem da área que atingisse $60,70,80,90,100,200,250$, e 300 lux em pelo menos $50 \%$ do tempo das horas analisadas, para vidro com três transmissões visíveis distintas (Tvs $88 \%$, Tvs $60 \%$ e Tvs $40 \%$ ). Para os ambientes que possuíam sacada, foram gerados os resultados de autonomia da luz natural para o ambiente isoladamente $e$ ao incorporar a área da sacada à sua área. Além disso, de cada um desses resultados acompanha a identificação da porcentagem de céu visível e obstruído.

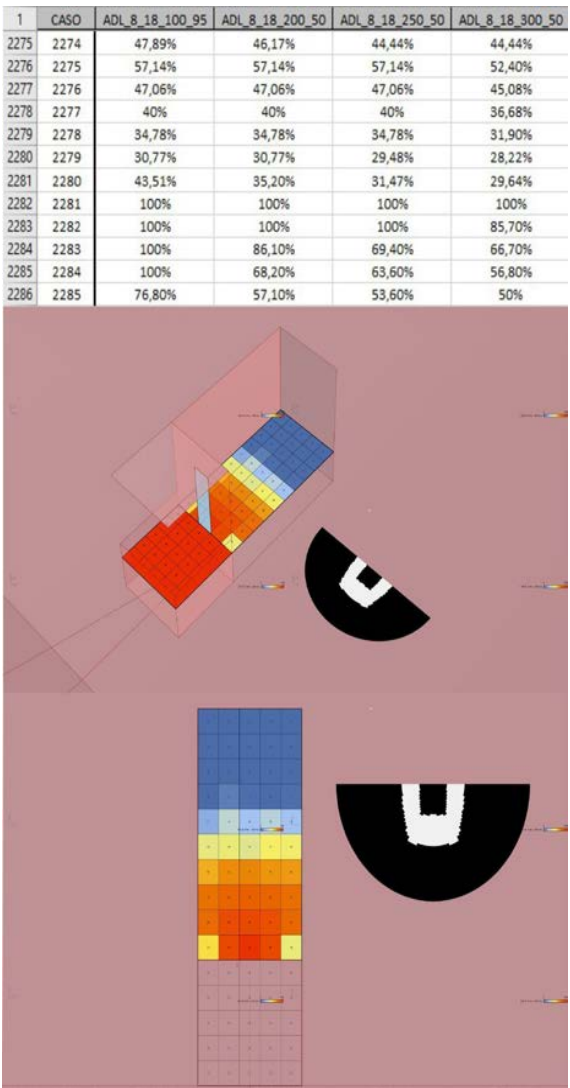

Figura 5: Os resultados do processo, dados com as simulações salvos em Excel e as imagens.

Ao final, um total de 2640 cenários variados foram simulados, resultando em total de mais de 23.760 arquivos de simulação, 47.520 imagens (jpg) e 427.680 resultados salvos em 18 planilhas de Excel. As simulações ocorreram em um intervalo de tempo de 5 meses, de novembro de 2019 a abril de 2020 , contando as rodadas de simulação e etapas de validação. 


\section{CONCLUSÃO}

O objetivo principal do artigo foi alcançado, uma vez que se desenvolveu e validou-se uma programação paramétrica autônoma capaz de repetir tarefas relacionadas a simulação e armazenamento de dados. Concluiu-se que mesmo providos de um aparelhamento tecnológico assertivo, os resultados finais necessitam de uma equipe de especialistas para a interpretação e validação dos resultados. Parte dos erros encontrados, durante e ao fim do processo, puderam ser identificados com o auxílio de interpretações humanas externas, que possuem uma garantia teórica e prática para uma previsão plausível do resultado. A construção do modelo permitiu observar que atribuímos uma confiabilidade aos resultados provenientes das ferramentas digitais, mas essas informações ainda precisam ser verificadas para obterem credibilidade. Pois mesmo se os processos apresentarem uma determinada autonomia, esses ainda são desenvolvidos por intermédio humano.

Os resultados provenientes do processo constituem uma base de dados robusta sobre o desempenho luminoso de edificações e são de múltiplas aplicações. Até o momento, a base de dados gerada já foi utilizada para a proposição de uma ferramenta simplificada para estimar o desempenho luminoso de edificações residenciais no contexto da revisão da norma de Desempenho de edificações habitacionais - ABNT NBR 15575:2013 e serão utilizados para a proposição de metamodelos preditivo com base em redes neurais artificiais. Ressalta-se que 0 processo como foi estruturado permite a ampliação da base de dados com facilidade, conforme demandas futuras.

\section{AGRADECIMENTOS}

Os autores agradecem aos integrantes do grupo de estudos do LABCON para a revisão do item de Desempenho Lumínico da Norma ABNT NBR 15.575 pela colaboração na etapa de validação do processo paramétrico. Parte desse trabalho teve auxílio do Conselho Nacional de Desenvolvimento Científico e Tecnológico CNPq [grant Brasil 151162/2019-0, grant CNPq - Brasil 307179/2016-8.

\section{REFERÊNCIAS}

ALSHOUBAKI, Hind et al. Innovative Design Strategy to Develop Facades Opening for a Commercial Building in Amman, Jordan. International Journal Of Applied Engineering Research, v. 11, n. 7, p.5288-5292, 2016.

BOYCE, P.r.. Illuminance Selection Based on Visual Performance-and other Fairy Stories. Journal Of The Illuminating Engineering Society, [s.I.], v. 25, n. 2, p.41-49, jul. 1996. Informa UK Limited. http://dx.doi.org/10.1080/00994480.1996.10748146.

CARTANA, Rafael Prado; PEREIRA, Fernando Oscar Ruttkay; BERTÉ, Eduardo João. AVALIAÇÃO DE DESEMPENHO TÉRMICO E LUMINICO DE ELEMENTOS DE CONTROLE SOLAR PROJETADOS ATRAVÉS DE MODELAGEM PARAMÉTRICA. Xvi Encontro Nacional de Tecnologia do Ambiente Construĺdo - Entac 2016, São Paulo, v. [1], n. [1], p.1-16, set. 2016

EMAMI, Niloufar; KHODADADI, Anahita; VON BUELOW, Peter. Design of a Shading Screen Inspired by Persian Geometric Patterns: An Integrated Structural and Daylighting
Performance Evaluation. lass-slte 2014, Brasilia, v. [1], n. [1], p.1-11, set. 2014.

DIVA for Rhino: Software for Buildings Daylight Analysis for. v. 2.0.2014.

FELIPPE, Alexandre Reis et al. Modelagem paramétrica para simulação do desempenho da iluminação natural e termoenergético da edificação. Sigradi Xix Congresso da Sociedade Ibero-americana de Gráfica Digital, Florianópolis, p.399-404, nov. 2015.

JAKUBIEC J. A. e REINHART, C. The Adaptive Zone - A Concept for Assessing Glare Throughout Daylit Spaces. In: Proceedings of Building Simulation, 12th Conference of International Building Performance Simulation Association. Sydney, 2011. 14-16 p.

MARIANO, Pedro Oscar Pizzetti; PEREIRA, Alice Theresinha Cybis; VAZ, Carlos Verzola. Avaliação luminosa de elementos de fachada com características fractais. Parc Pesquisa em Arquitetura e Construção, [s.I.], v. 9, n. 1, p.3-18, 31 mar. 2018. Universidade Estadual de Campinas. http://dx.doi.org/10.20396/parc.v9i1.8650255.

MARIANO, Pedro Oscar Pizzetti. PROCESSO DE PROJETO PARAMÉTRICO DE ELEMENTOS DE FACHADA COM CARACTERISTICAS DA GEOMETRIA FRACTAL CON. 2018. 189 f. Dissertação (Mestrado) - Curso de Arquitetura e Urbanismo, Projeto e Tecnologia do Ambiente Construído, Universidade Federal de Santa Catarina, Florianópolis, 2018.

Mardaljevic, J. and Christoffersen, J., 'Climate connectivity' in the daylight factor basis of building standards. Building and Environment; 2017,113: 200-209. https://doi.org/10.1016/j.buildenv.2016.08.009

Mcneel, R.; Associates. Rhinoceros 3D modelling Software.v. 5 2014

OXMAN, Rivka. Theory and design in the first digital age. Design Studies, [s.I.], v. 27, n. 3, p.229-265, maio 2006. Elsevier BV. http://dx.doi.org/10.1016/j.destud.2005.11.002.

OXMAN, Rivka. 'DIGITAL DESIGN THINKING': IN THE NEW DESIGN IS THE NEW PEDAGOGY. Caadria 11, Kumamoto, Japão, v. [1], p.37-46, 2006.

OXMAN, Rivka; HAMMER, Roey; ARI, Shoham Ben. Performative Design in Architecture: Employment of Virtual Prototyping as a Simulation Environment in Design Generation. Ecaade 25, Frankfurt, v. [1], n. [1], p.227-234, 2007.

REINHART, Christoph F.; MARDALJEVIC, John; ROGERS, Zack. Dynamic Daylight Performance Metrics for Sustainable Building Design. Leukos, [s.1.], v. 3, n. 1, p.7-31, jul. 2006.

RUTTEN, David; MCNEEL, Robert \& Associates, 2014. Grasshopper 3D, EULA. www.grasshopper3d.com.

ROUDSARI, Mostapha Sadeghipour; PAK, Michelle, 2013. Ladybug: a parametric environmental plugin for grasshopper to help designers create an environmentally-conscious design. In: Proceedings of the 13th International IBPSA Conference Held in Lyon, France Aug 25-30th. (http://www.ibpsa.org/proceedings/BS2013/p_2499.pdf).

Tregenza, P.R.; Waters, I. Daylight coefficients. Lighting Research and Technology; 1983, 15: 65-71. https://doi.org/10.1177/096032718301500201.

Ward, G. J. The RADIANCE Lighting Simulation and Rendering System. In: Computer Graphics - SIGGRAPH Conference. Orlando/Flórida. Jul. 1994; 459-72.

WIRZ-Justice, A., FOURNIER, C. (2010) Light, Health and Wellbeing: Implications from chronobiology for architectural design, World Health Design, vol. 3. 